\title{
THE IMPACT OF NETWORK ARCHITECTURE ON \\ DATA ACQUISITION SYSTEMS
}

\author{
Daniel S. Skelley \\ Naval Air Warfare Center Aircraft Division \\ Sidney R. Jones, Jr. \\ Naval Air Warfare Center Aircraft Division
}

\begin{abstract}
The purpose of this paper is to present a broad view of the impact of network architectures on future data acquisition systems. The major advantages and challenges associated with the use of network architectures are rooted in the packetized structure of the data. Many of the issues raised are subtle and complex. It is not the intent of this paper to give these issues the thorough academic and technical analysis they deserve. It is the hope of the authors this paper will generate awareness and discussion on these issues.
\end{abstract}

\section{KEY WORDS}

Instrumentation, data acquisition, networks, protocols, standards.

\section{INTRODUCTION}

The Department of Defense (DoD) uses a wide variety of data acquisition systems to provide data in support of the test and evaluation of weapon systems. Typically, these are synchronous time division multiplexed (TDM) systems distributed throughout the test article. The various components of the system are connected via a synchronous instrumentation bus of which there are several in use today. Present data requirements exceed the capacity of these busses. Emerging requirements for the fusion of multiple high speed data sources (high speed video, avionics, etc.) further establish the inadequacy of current instrumentation bus speeds. In response to these requirements, vendors are currently developing very fast, very capable, instrumentation products (e.g., solid-state recorders). A new high-speed instrumentation bus standard, with the bit rate to allow these products to fulfill their potential, is required. An open standard would allow vendors to develop products that can be used in many applications and thus achieve broad market 
appeal. This would foster competition, broaden purchasing options, and allow interoperability of different vendors' components.

Existing and near horizon commercial communication standards are showing promise as a potential high -speed instrumentation bus standard. In recognition of this, the Office of the Secretary of Defense (OSD) sponsored the Next Generation Instrumentation Bus (NexGenBus) project. The goal of the NexGenBus project is to adopt an open, commercial communications standard as a future instrumentation bus (reference 1). Working closely with the NexGenBus project, the Telemetry Group of the Range Commander's Council (RCC) is pursuing a similar and complimentary goal. These two efforts have the potential to drastically change the architecture of future data acquisition systems.

The OSD and RCC efforts have engineering support from the Army, Air Force, and Navy. These efforts have defined the future instrumentation bus requirements and conducted an evaluation of commercial standards with respect to their ability to meet those requirements. A worldwide search yielded in excess of 30 commercial communication standards. Initial evaluations indicate that three of these standards are viable candidates as a next generation instrumentation bus. These standards are fast (bus speeds from 400 megabits tolgigabit per second) and are network-based communication busses that utilize packetized data structures.

Users and producers of instrumentation hardware are beginning to discuss the implications of using a data acquisition network that employs communication standards and packetized data. A network-based communications bus would be used to interconnect the various components of a data acquisition system to form the data acquisition network. This data acquisition network is much more than just a distributed data acquisition system. The data acquisition network will use packetized data, protocols, and architectures similar to traditional computer networks (e.g., Ethernet). On a limited scale, data acquisition networks are currently being designed. In early1998, the Navy took delivery of a wireless data acquisition system. This system's components communicate via a wireless local area network utilizing packetized data. The system has not been used in an actual flight test environment; however, the test results are promising.

\section{DATA ACQUISITION NETWORKS}

The use of data acquisition networks will have a ripple effect throughout the entire test and evaluation range structure. Figure 1 illustrates how data acquisition networks will initially be used. 

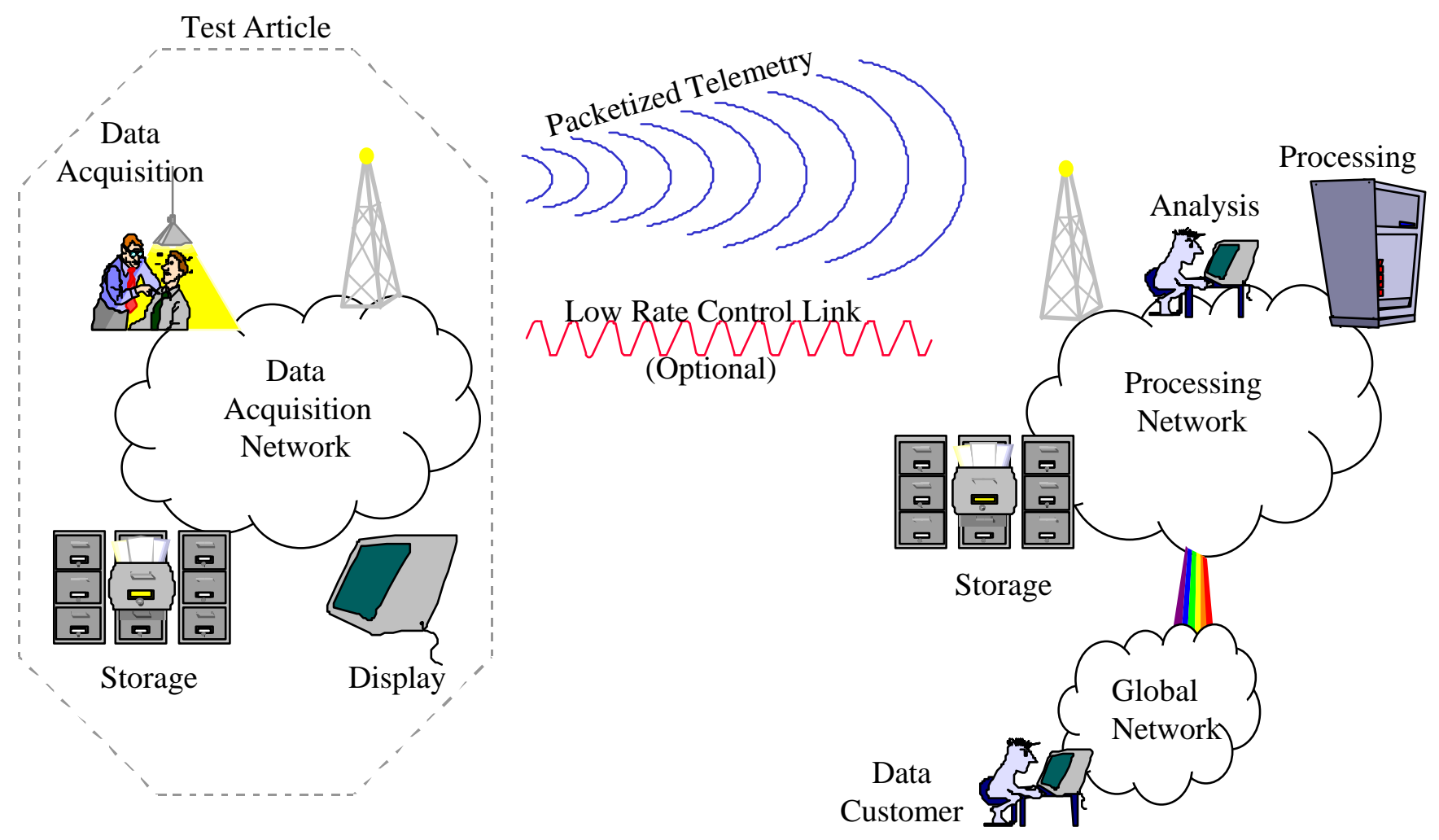

Figure 1. Telemetry System Utilizing a Data Acquisition Network

Data is collected, formatted into packets, stored, displayed, and/or transmitted by the data acquisition network. The telemetry subsystem reformats the data (if necessary) and transmits the data to the processing network, via a packetized telemetry link. Data packets are received and placed on the processing network for analysis, distribution, and display. In its earliest and simplest form, the RF link would be a one-way link. A sophistication of this approach would be the addition of a low rate control link. The low rate control link would provide a moderate level of remote control of the data acquisition network. Items such as the telemetry frequency and the data sets being transmitted could be controlled via this link. The control link and other advanced telemetry concepts are being investigated by the Advanced Range Telemetry program (references 2 and 3).

Data acquisition networks have numerous advantages and challenges. Many of these are extremely obvious, others are quite subtle. Understanding the advantages and addressing the challenges will ensure the effective utilization of data acquisition networks.

\section{ADVANTAGES OF DATA ACQUISITION NETWORKS}

The network technology market has made significant gains in performance and efficiencies over the last few years. Network speeds have increased dramatically and the associated hardware has become smaller and less expensive. Global networks are moving 
unprecedented volumes of data and information around the world. Open standards are guiding the physical and logical infrastructure of networks of all sizes. The T\&E community must take advantage of these trends today, to build the required capability for tomorrow.

Rapidly changing technology and acquisition reform have led DoD to rely more heavily on commercial off the shelf (COTS) hardware and software. The use of data acquisition networks, based on commercial standards, strongly supports the use of COTS hardware and software. The technical and cost benefits gained by leveraging the electronics industry investment in network technology are significant. Industry invests a tremendous amount of time, money, and talent in the development of commercial standards and associated products. DoD does not have the resources to duplicate these efforts. The availability of widely used state of the art equipment, designed to commercial standards is an opportunity that cannot be ignored.

The fundamental technical and operational benefit of network based data acquisition systems is the packet structure of the data. We live in a networked world, data is not sent around the globe in major and minor frames constructed according to IRIG 106 (reference 4). It is sent in packets. A traditional TDM data stream, stuffed into packets, and sent over ground-based networks is clumsy at best. The time has come for the data acquisition system to format its data directly into packets. Packetized data can be routed via groundbased networks to worldwide customers with ease.

The opportunities provided by full network connectivity of all components of the data acquisition system are complex and far reaching. In a traditional data acquisition system, all data acquisition units (DAUs) send their data to a central controller. The central controller then formats the data into a TDM data stream and routes the data to a recorder and/or transmitter. In a data acquisition network, any DAU can send data to any other DAU. This allows easy implementation of functions such as system wide data driven acquisition or DAU produced calculated parameters. Any or all DAUs on the network can change their data acquisition strategy or produce a calculated parameter based on any data available on the network. The opportunities opened up by full network connectivity are only limited by TDM paradigms.

The commercial communication standards being considered are fast - very fast. While speed is not a function of network architecture, the speed at which these networks operate is certainly an advantage. Data acquisition options and strategies not previously considered are now possible. Given these speeds, the emerging requirement for the fusion and correlation of multiple high-speed data sources (e.g., digitized video, high-speed avionics busses, and analog data) can be met. The speeds at which these standards 
operate provide bus bandwidth well in excess of current and near-term requirements. This will ensure a long useful life for the next generation instrumentation bus.

There are many advantages to using data acquisition networks. Some prominent examples include leveraging the industry's knowledge, investment and high production quantities, compatibility with global networks through packetized data, and open commercial standards. Any of these examples make a compelling case for the use of data acquisition networks. Taken as a whole, the case is overwhelming.

\section{CHALLENGES OF DATA ACQUISITION NETWORKS}

The introduction of new technologies typically involves challenges; data acquisition networks are no exception. Many of these challenges are complex and should not be trivialized. The packet structure of the data is the basis of many of the most critical challenges. Numerous factors that are taken for granted in traditional TDM systems (such as time correlation, simultaneous sampling, latency, extremely low probability of lost data, etc.) become challenges when the use of data acquisition networks are considered.

Packetized data structures are one of the biggest challenges to the implementation, operation, and management of network based data acquisition. The switch from TDM data to packetized data will challenge traditional data processing systems. In general, TDM systems are synchronous and deterministic. Each data word and its time relationship to other words in the TDM stream are clearly defined. The synchronous and deterministic characteristics of TDM data are exploited by the algorithms of most data processing systems. In comparison to TDM data, the time relationship and definition of packetized data are more complex. For example, in some systems, it is possible for packetized data to be delivered out of order and the data structures within the data packets may be dynamic. These types of issues will create challenges that must be dealt with in the data processing algorithms.

Latency management and the associated probability of delivery are another source of challenges facing the use of data acquisition networks. Synchronous TDM data has a constant latency with a very high (almost certain) probability of delivery. Constant latency can be compensated for in data processing algorithms. In contrast, the latencies within a communications network are dynamic and vary over a wide range. In some networks, data may never be delivered. Without latency management and data delivery guarantees, networks cannot be used effectively for data acquisition. Some networks have Quality of Service (QoS) features that manage these types of issues. Managing latency and guaranteeing delivery through some form of QoS are critical issues facing the use of data acquisition networks. 
Packetized data, by its very nature, can be bandwidth inefficient. Using identical data sets, packetized data will require more bandwidth than an equivalent TDM stream. This is a significant issue when RF transmission of the data is considered. The amount of spectrum available to DoD for transmission of test data has been decreasing. This trend is expected to continue into the future. To compensate, raw data transmission must be replaced by the transmission of information. This would involve the application of algorithms that would limit the transmission of redundant data. The Advanced Range Telemetry program is actively pursuing this and other related concepts (references 2 and 3 ).

System wide simultaneous sampling is required for complex algorithms that combine multiple samples into a single derived parameter. Due to the asynchronous nature of traditional networks, system wide simultaneous sampling is a challenge. Simultaneous sampling is typically accomplished in one of two ways. The first requires a sample command to be sent to all nodes at the same time (i.e., low latency). The second requires a high degree of synchronization between the nodes which would allow all nodes to sample simultaneously. Commercial networks do not typically operate at the latency or synchronization levels that would be required. Any network-based instrumentation standard must address this issue.

Unique operating environments pose a challenge to the use of any COTS equipment. DoD applications for data acquisition networks will typically require operation in extreme environmental conditions. These include factors such as electromagnetic interference, temperature, vibration, condensation, and salt spray. The physical components (cables, connectors, etc.) of most commercial communication standards are not robust enough to handle these extreme factors. Use of a commercial standard may involve modifications to obtain the required environmental tolerances.

The challenges facing the widespread use of data acquisition networks are complex but solvable. However, a failure to recognize and deal with them could fragment the instrumentation community's use of this promising technology. The government, industry, and academia must work together. Careful thought, planning, and cooperation will ensure these challenges are met.

\section{THE FUTURE}

Members of the telemetry community are beginning to explore the possibility of telemetry networks. The key distinction between a telemetry network and the telemetry system discussed previously (figure 1) is the RF link. The unidirectional telemetry link is replaced with full two-way network connectivity. The ramifications of this change are extremely complex and far reaching. A thorough discussion of the telemetry network is beyond the 
scope of this paper. However, a high-level discussion is beneficial to understand how data acquisition networks would be utilized within the telemetry network.

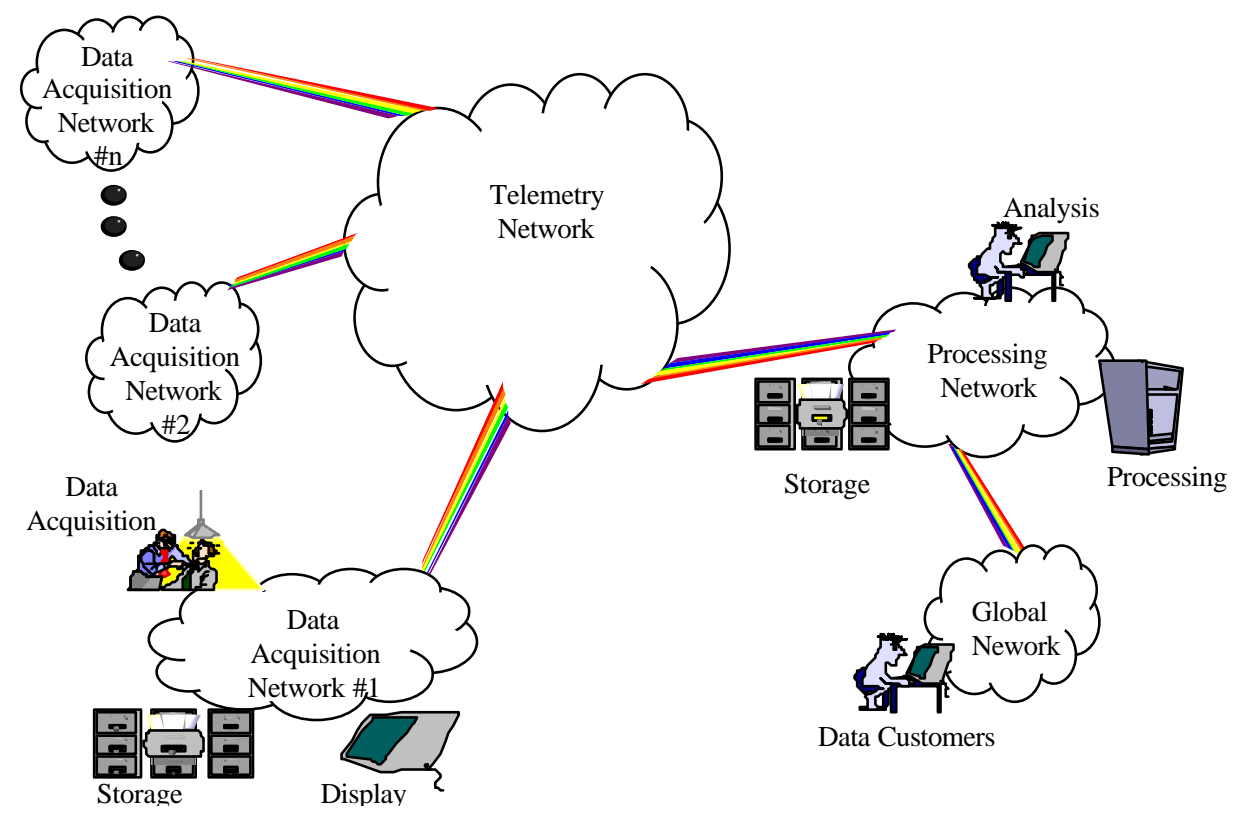

Figure 2. Telemetry Network (conceptual)

In the telemetry network, as envisioned by figure 2 , network connectivity would extend from the data acquisition network (on the test article) to the user. The data acquisition network logs onto the telemetry network at the start of the test. Numerous data acquisition networks may be logged onto the telemetry network at any one time. Data is acquired, packetized, and sent through the telemetry network to any authorized user. This is a dynamic process with data packets being requested and defined throughout test execution. An authorized user, anywhere in the world, could remotely log onto the telemetry network and request and receive test data. The futuristic telemetry network presented here is based on the Air Force Real Time Telemetry Network (RTTN) proposal (reference 5).

There are mind-boggling challenges to implementing and managing the telemetry network. Protocols and architectures must be defined. Where possible, these protocols and architectures should be based on commercial communications standards. Network management issues such as QoS, authorized users, prioritization, data security, and bandwidth allocation need to be addressed. In the future (albeit distant future), when the telemetry network becomes a reality, the data acquisition network will achieve its full promise of enabling seamless connectivity from the DAU (on the test article) to the user (anywhere in the world). 


\section{CONCLUSION}

The benefits to using data acquisition networks are real and significant. The compatibility of packetized data with the large installed infrastructure of networks will provide significant advantages. The challenges to implementing data acquisition networks can be overcome. Traditional TDM systems will be replaced by data acquisition networks as these challenges are solved. Technology trends, the RCC task, and the NexGenBus project, provide little doubt that widespread use of data acquisition networks are on the near horizon.

Data acquisition networks will usher in a new era in instrumentation design, affordability, performance, and complexity.

\section{ACKNOWLEDGEMENTS}

The tri-service members of the RCC task and the NexGenBus project are leading the way to data acquisition networks. Many of the thoughts and ideas presented in this paper had their genesis in lively conversations with these folks.

\section{REFERENCES}

1. D. Skelley, "Next Generation Instrumentation Bus (NexGenBus); The Program, The Potential, The Challenges," Proceedings of the International Test and Evaluation Workshop, http://www.edwards.af.mil/itea/papers98.htm, Lancaster, CA, March 2326, 1998.

2. C. Irving, "Advanced Range Telemetry," Proceedings of the International Test \& Evaluation Workshop, http://www.edwards.af.mil/itea/papers98.htm, Lancaster, CA, March 23-26, 1998..

3. T. Chalfant, C. Irving, "Range Telemetry Improvement and Modernization," Proceedings of the International Telemetry Conference, Volume XXXIII, Las Vegas, NV, October 27-30, 1997.

4. Telemetry Group Range Commanders Council, "IRIG Standard 106-96 Telemetry Standards," Secretariat, Range Commanders Council, U.S. Army White Sands Missile Range, NM, May 1996C.

5. T. Chalfant, R. Grahn, “Airborne Instrumentation How Will It Play In The Future," Proceedings of the International Test \& Evaluation Workshop, http://www.edwards.af.mil/itea/papers98.htm, Lancaster, CA, March 23-26, 1998. 
6. "Welcome to ARTM - Advanced Range Telemetry," http://www.elan.af.mil/artm.

7. "NexGenBus Home Page," April 15, 1998, http://nexgenbus.nawcad.navy.mil. 